\title{
Comparación entre los diagramas TTS obtenidos mediante los ensayos normalizados Huey y Strauss modificado para el acero inoxidable AISI $304^{(*)}$
}

\author{
E. Otero ${ }^{(* *)}$, A. Pardo ${ }^{(* *)}$, E. Sáenz ${ }^{(*)}$, M.V. Utrilla ${ }^{(* *)}$ y F.J. Rodríguez-Bermejo ${ }^{(* *)}$
}

Resumen Se comparan los diagramas Temperatura-Tiempo-Sensibilización TTS, obtenidos mediante los ensayos normalizados de corrosión intergranular Huey y Strauss modificado, que, respectivamente, utilizan electrólitos fuerte y suavemente oxidantes, mostrándose que los diagramas obtenidos son diferentes. Si una muestra evaluada mediante el ensayo Strauss modificado se encuentra sensibilizada, entonces lo estará también en el ensayo Huey, pero no necesariamente ocurrirá lo contrario; esta diferencia se asocia a la necesidad de la existencia de una zona continua empobrecida en cromo a lo largo del límite de grano para que el material se sensibilice con respecto al ensayo Strauss modificado, condición innecesaria para el caso del ensayo Huey.

Palabras clave: Aceros inoxidables austeníticos. Corrosión intergranular. Ensayo Huey. Ensayo Strauss modificado. Sensibilización.

\section{Comparison between TTS diagrams obtained by Huey and modified Strauss standard tests for AISI 304 stainless steel}

\begin{abstract}
This work presents a comparison of Temperature-Time-Sensitization TTS diagrams obtained by Huey and modified Strauss standard, for detecting susceptibility to intergranular attack. The difference between these tests is the use of a strong or a slighty smooth oxidizing electrolite. The diagrams obtained are different and if a sample tested by modified Strauss is sensitised, then it will be sensitised in the Huey test, but the contrary is not always true. This difference is because a sensitised sample has to have a continuous band lacking in chromiun along the grain boundary in order to be sensitised in the modified Strauss test. This condition is not necessary in the Huey test.
\end{abstract}

Keywords: Austenitic stainless steel. Intergranular corrosion. Huey test. Modified Strauss test. Sensitisation.

\section{INTRODUCCIÓN}

El acero inoxidable AISI 304 es una aleación austenítica que al someterse a un determinado tratamiento térmico presentará, al exponerse a un medio agresivo, fenómenos de corrosión localizada en límite de grano, denominada corrosión intergranular (CI).

$(\bullet)$ Trabajo recibido el día 26 de junio de 1995

(*) Lab. de Corrosión. Facultad de Ciencias e Ingeniería. Pontificia Universidad Católica del Perú. Lima (Perú).

(**) Dpto. de Ciencia de Materiales e Ingeniería Metalúrgica. Facultad de Ciencias Químicas. Universidad Complutense. 28040-Madrid (España).
Hasta el momento no existe ninguna teoría que explique, para todos los medios agresivos, el fenómeno de la CI (1), existiendo diversas hipótesis que justifican el fenómeno en cada caso y para los diversos medios agresivos (2-4). Levin (5), tras estudiar distintas hipótesis, menciona que solamente tres de las teorías expuestas son las fundamentales para explicar los fenómenos de la CI (empobrecimiento en cromo, disolución de fases precipitadas y elevados esfuerzos en límite de grano por la precipitación de fases). Hänninen (6) sugirió, tras una revisión de artículos relacionados con la $\mathrm{CI}$, que en medios suavemente oxidantes, como el ensayo Strauss, el empobrecimiento en cromo es el mecanismo predominante, mientras que en medios 
fuertemente oxidantes, como el ensayo Huey, la segregación de impurezas debe ser el factor predominante.

Abe y Kaneko (7 y 8), a partir de la revisión de Hänninen (6) y de sus propios resultados, propusieron una teoría unificada para justificar los mecanismos de la CI en los aceros inoxidables, la cual se engloba dentro de dos mecanismos dependientes de la composición (empobrecimiento en cromo ( 9 y 10), segregación de elementos en límite de grano (11 y 12)) y de la presencia de componentes intermetálicos en límite de grano ( 7,8 y 13).

Para mostrar los efectos que el tratamiento térmico tiene en el material al ser expuesto a un medio agresivo, se utiliza la curva denominada TTS (Temperatura-Tiempo-Sensibilización); en ella se delimitan las condiciones de temperatura y tiempo para las cuales se produce la sensibilización y, por lo tanto, se produce la CI cuando posteriormente el material entra en contacto con determinados medios agresivos. Cualquier tratamiento térmico que quede en el exterior de esta curva no provocará sensibilización del acero.

Una de las formas de evaluar la susceptibilidad de los aceros inoxidables al ataque intergranular, consiste en la realización de ensayos químicos normalizados. Dentro de estos ensayos se encuentran el Huey y el Strauss modificado. En este último, el ataque se localiza en las zonas empobrecidas en cromo, mientras que en el ensayo Huey, además de atacarse esas regiones, se atacan los precipitados presentes en la matriz, tales como carburos, fase $\sigma$, fases de transición de molibdeno, de fósforo y de sus compuestos, además de distintas zonas de segregación (14-17). El ensayo más extendido, al menos para evaluaciones en la industria, para detectar la susceptibilidad al ataque intergranular, es el Strauss modificado, utilizándose el ensayo Huey en la evaluación de materiales que estarán expuestos al ácido nítrico.

En este estudio se comparan las curvas TTS, obtenidas para el acero AISI 304, mediante los ensayos Huey y Strauss modificado, mostrándose las principales diferencias entre ambas curvas. Además, se realiza un estudio microestructural del acero con objeto de entender la diferente respuesta del material sensibilizado en estos ensayos.

\section{MÉTODO EXPERIMENTAL}

Se utilizó, como material de estudio, acero inoxidable austenítico AISI 304, obtenido por colada y laminación en caliente, de uso común en la industria. La composición química de este material es, en porcentaje en masa: $0,042 \mathrm{C}, 18,3 \mathrm{Cr}, 8,05 \mathrm{Ni}, 1,39$ Mn, 0,348 Si, 0,28 Mo, 0,028 P, 0,002 S y 0,06 N.

Las muestras se prepararon a partir de una chapa de 2,0 $\mathrm{mm}$ de espesor. Las dimensiones de las mis- mas eran $15 \times 15 \times 2 \mathrm{~mm}$ para el ensayo Huey y $25 \times 75 \times 2 \mathrm{~mm}$ para el ensayo Strauss modificado, correspondiendo la mayor de las dimensiones con la dirección de laminado.

Todas las muestras se sometieron, inicialmente, a un tratamiento térmico de solubilización a $1.573 \mathrm{~K}$ durante $10 \mathrm{~min}$, con temple posterior en agua, para disolver los posibles carburos presentes en la matriz.

Con el fin de introducir cambios microestructurales, relacionados fundamentalmente con la precipitación de carburos ricos en cromo, se efectuaron tratamientos térmicos de sensibilización en el intervalo de temperaturas comprendido entre 773-1.173 $\mathrm{K}$ y $823-1.073 \mathrm{~K}$, y tiempos entre $1-400 \mathrm{~min}$ y $30-$ $1.000 \mathrm{~min}$, para los ensayos Huey y Strauss modificado, respectivamente.

Las muestras, antes de ensayarse, se desbastaron sucesivamente con papel de carburo de silicio de granulometría 80 y 120 , con objeto de obtener un acabado superficial uniforme y de eliminar cualquier resto de óxido en superficie generado durante el tratamiento térmico. El desbaste se realizó en medio acuoso y, posteriormente, se limpiaron con agua corriente y alcohol en baño de ultrasonidos.

Una vez preparadas, utilizando un pie de rey (calibre con aproximación de $0,05 \mathrm{~mm}$ ), se determinó la superficie total de cada muestra, desengrasándose finalmente con acetona y quedando listas para los ensayos que se describen a continuación.

\subsection{Ensayo Huey}

Este ensayo se recoge en la norma ASTM A262 , práctica C (18), consistente en exponer las muestras en ácido nítrico al $65 \%$ en ebullición por un período total de cinco ciclos de $48 \mathrm{~h}$ cada uno, renovándose en cada ciclo el ácido. La cantidad de ácido sugerida por la norma es de por lo menos 20 $\mathrm{ml} / \mathrm{cm}^{2}$ de superficie expuesta; en los ensayos realizados se utilizó, aproximadamente, un $50 \%$ más de la cantidad mínima recomendada.

Al finalizar cada ciclo se limpiaron las muestras con agua y jabón y, posteriormente, con acetona en baño de ultrasonidos, para luego determinar su peso y la velocidad de corrosión $\left(V_{\text {corr }}\right)$ mediante la expresión:

$$
V_{\text {corr }}=728,98 \mathrm{~W} /(A \cdot d \cdot t)(\mathrm{cm} / \mathrm{mes})
$$

donde: $W=$ Pérdida de peso, $\mathrm{g}$

$$
\begin{aligned}
A= & \text { Superficie expuesta de la muestra, } \mathrm{cm}^{2} \\
t= & \text { Tiempo de exposición, } \mathrm{h} \\
d= & \text { Densidad de la muestra }\left(7,9 \mathrm{~g} / \mathrm{cm}^{3}\right. \\
& \text { para el AISI 304) }
\end{aligned}
$$

Todas las muestras se ensayaron de forma individual, para evitar una posible interferencia entre 
las mismas por la posible presencia de cationes disueltos. La $V_{\text {corr }}$ final es el promedio de las $V_{\text {corr }}$ obtenidas en cada ciclo.

Se considera que el material se encuentra sensibilizado a la corrosión intergranular si la $V_{\text {corr }}$ $>0,0037 \mathrm{~cm} / \mathrm{mes}$, criterio propuesto para el acero AISI 304 por distintos autores (19 y 20).

\subsection{Ensayo Strauss modificado}

Este ensayo se recoge en la norma ASTM A-262, práctica E (21) y se conoce como ensayo Strauss modificado o acelerado. Se emplea, fundamentalmente, para medir la susceptibilidad al ataque intergranular asociado a la existencia de zonas empobrecidas en cromo en el límite de grano, por la precipitación de fases ricas en cromo (22).

El ensayo comprende dos partes: la primera consiste en exponer la muestra en una disolución de sulfato de cobre y ácido sulfúrico en ebullición durante $24 \mathrm{~h}$, en presencia de viruta de cobre. Posteriormente, las probetas se doblan hasta un ángulo de $180^{\circ}$ y se clasifican como sensibilizadas (no aceptables), o no sensibilizadas (aceptables), dependiendo de si la superficie central de la zona de doblado se encuentra agrietada o libre de grietas, por lo que este ensayo es de tipo cualitativo.

La disolución de ensayo se prepara disolviendo $100 \mathrm{~g}$ de $\mathrm{CuSO}_{4} \cdot 5 \mathrm{H}_{2} \mathrm{O}$ en $700 \mathrm{ml}$ de agua destilada, añadiendo, a continuación, $100 \mathrm{ml} \mathrm{de} \mathrm{H}_{2} \mathrm{SO}_{4}$ con una densidad de $1,84 \mathrm{~g} / \mathrm{cm}^{3} \mathrm{y}$, finalmente, se diluye hasta 1 litro de agua destilada. La norma exige un mínimo de $8 \mathrm{ml}$ de disolución $/ \mathrm{cm}^{2}$ de superficie expuesta. En este estudio se utilizó aproximadamente un $70 \%$ más, para tener la garantía de que la muestra y toda la viruta de cobre se encuentra sumergida durante el ensayo. Todas las muestras se ensayaron de forma individual.

Para la realización del ensayo de doblado se empleó una máquina universal de tracción Mohr Federhaf Losenhause (MFL), cuya carga máxima es de 40 t. El doblado se realizó utilizando un mandril de diámetro igual al espesor de la muestra, siendo el eje de doblado perpendicular a la dirección de laminado. La velocidad de aplicación del punzón fue de $1 \mathrm{~mm} / \mathrm{s}$, aproximadamente. Para evitar que el soporte del mandril deformara las probetas, fue necesario limitar el recorrido de la máquina, quedando las probetas dobladas hasta un ángulo de aproximadamente $120^{\circ}$. El doblado hasta $180^{\circ}$ se completó, posteriormente, utilizando los platos de compresión de la misma máquina.

\subsection{Estudio microestructural}

El objetivo es estudiar la microestructura de las muestras y su influencia en la respuesta a los dos tipos de ensayo. El análisis se ha centrado, fundamentalmente, en la presencia de precipitados en límite de grano y en la forma, tamaño y distribución de estos carburos como consecuencia del tratamiento de sensibilización. El reactivo utilizado es el denominado "glicerregia", cuya composición química, en partes por volumen (ppv), es:

$$
\begin{aligned}
& -1 \text { ppv de glicerina o metanol } \\
& -1 \mathrm{ppv} \text { de } \mathrm{HNO}_{3} \text { al } 60 \% \\
& -3 \mathrm{ppv} \text { de } \mathrm{HCl} \text { al } 35 \%
\end{aligned}
$$

Las muestras sometidas a ensayos de inmersión quedan recubiertas por productos de corrosión no conductores, por lo que es necesario depositar sobre ellas, mediante sputtering, una fina película conductora de oro.

El estudio metalográfico se realizó mediante microscopía electrónica de barrido (SEM), utilizándose un microscopio electrónico JEOL-JSM-35C, de $6 \AA$ de resolución.

\section{RESULTADOS}

La figura 1 muestra la microestructura del acero AISI 304, objeto de estudio. Se observa una estructura monofásica austenítica, con los límites de grano bien definidos y con presencia de maclas características de estructuras cúbicas centradas en las caras.

\subsection{Resultados obtenidos con el ensayo Huey}

Para el acero en estado de solubilización, se obtuvo una $V_{\text {corr }}=0,0026 \mathrm{~cm} / \mathrm{mes}$, por lo que, partiendo de los criterios expuestos, el material no se

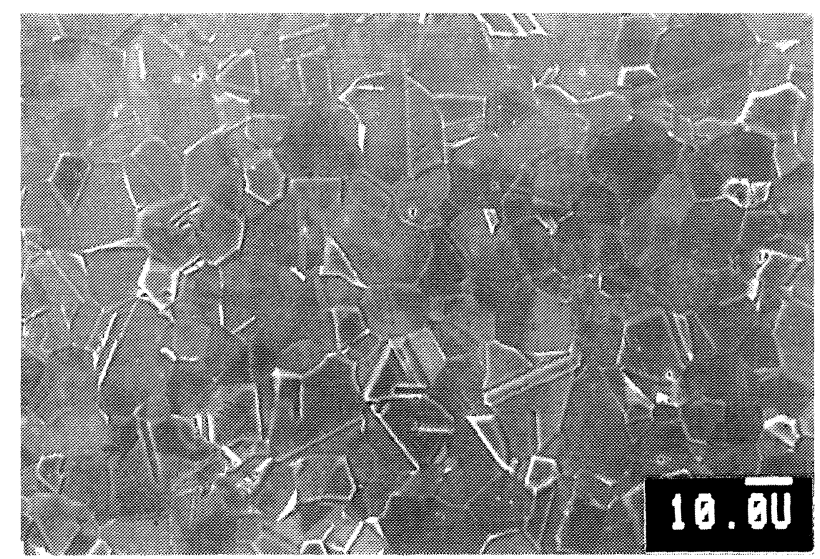

FIG. 1.- Microestructura de un acero AISI 304, solubilizado (tamaño de grano ASTM 9). $\times 600$.

Fig. 1.- Microstructure of AISI 304 stainless steel after solution treatment (ASTM 9 grain size). $\times 600$. 
encuentra sensibilizado a la corrosión intergranular. $\mathrm{El}$ ataque es de tipo superficial y no progresa hacia el interior de la muestra, localizándose principalmente en límites de grano.

La figura 2 muestra la $V_{\text {corr }}$ en función del ciclo de ensayo para condiciones de tratamiento térmico que producen o no sensibilización. Para todos los casos donde el material presenta sensibilización, la $V_{\text {corr }}$ aumenta con el tiempo de ensayo. El mencionado comportamiento permite que, en el caso de materiales con un fuerte grado de sensibilización, es decir, con elevadas $V_{\text {corr }}$, no sea necesario finalizar los cinco ciclos de ensayo para determinar el diagrama TTS.

En la figura 3, se muestra la $V_{\text {corr }}$ en función del tiempo y de la temperatura de tratamiento. Se puede observar que a determinadas temperaturas la tendencia de las curvas es similar a las homólogas de nucleación y crecimiento, hecho que se asocia a la cinética de precipitación de carburos en límites de grano. En el intervalo de temperaturas desde 873 hasta $973 \mathrm{~K}$, se observa un fuerte aumento en la $V_{\text {corr }}$, mientras que a mayores temperaturas el aumento es más suave. A temperaturas comprendidas entre 1.023 y $1.098 \mathrm{~K}$, se observa, inicialmente, un aumento en la $V_{\text {corr }}$ que disminuye al aumentar el tiempo de sensibilización, presentándose el fenómeno de desensibilización en el que la $V_{\text {corr }}$ disminuirá hasta valores inferiores al de sensibilización.

Una microestructura típica de material no sensibilizado se muestra en la figura 4a), observándose que el material sufre ataque superficial en límites de grano y que no progresa hacia el interior de la muestra.

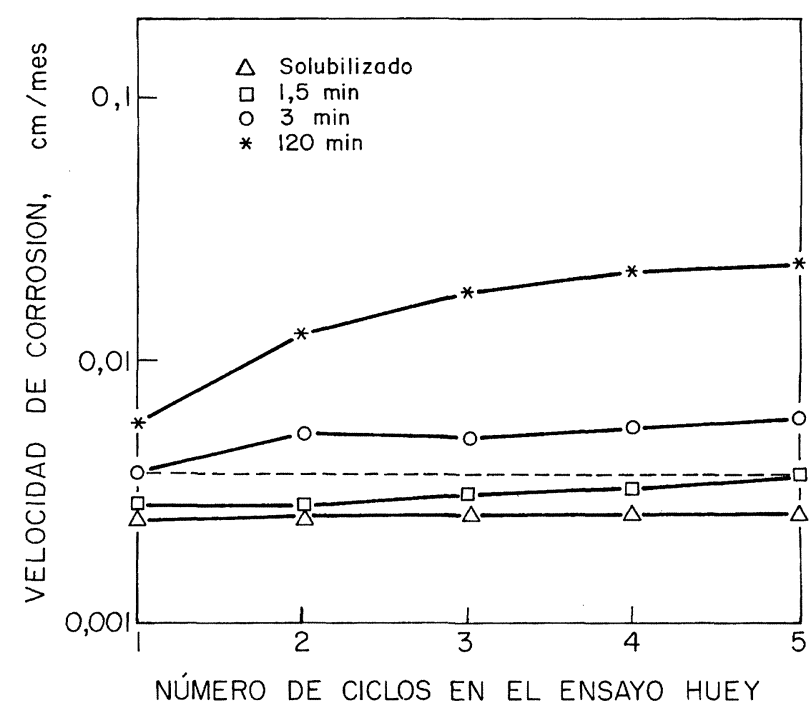

Fig. 2.- Velocidad de corrosión en función del ciclo de ensayo Huey, para un acero AISI 304 tratado térmicamente a $973 \mathrm{~K}$.

Fig. 2.- Corrosion rate as a function of the Huey cycle number, for AISI 304 stainless steel heat treated at $973 \mathrm{~K}$.

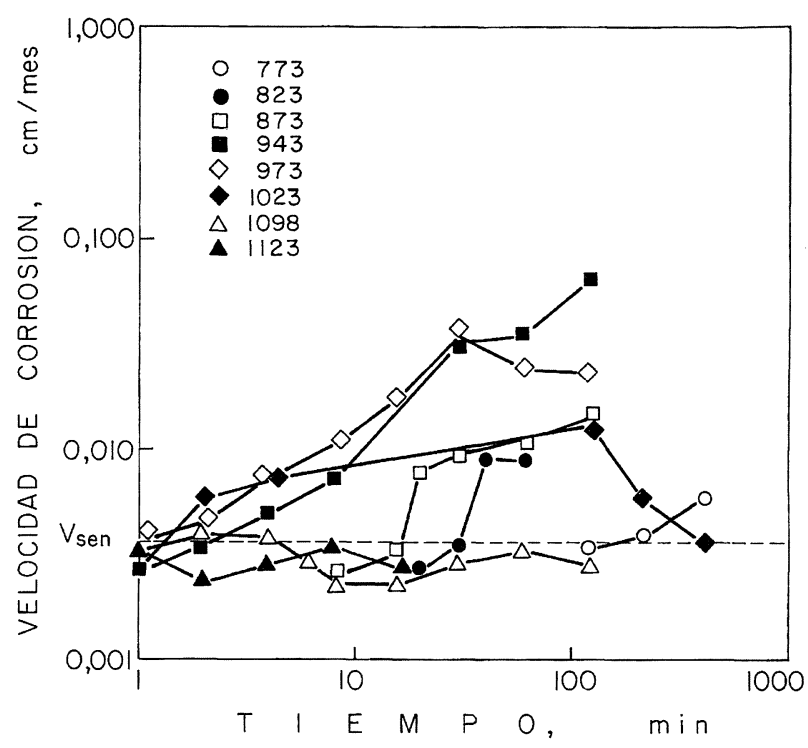

FIG. 3.- Velocidad de corrosión en función del tratamiento térmico, evaluada mediante el ensayo Huey, para un acero AISI 304.

Fig. 3.- Corrosion rate as a function of heat treatment for AISI 304, obtained from the Huey test.

En el caso de los materiales sensibilizados, el ataque es más acusado en límite de grano, progresando hacia el interior del material. Para elevadas $V_{\text {corr }}$, el material se descohesiona y llega a producirse, en determinadas áreas, desprendimiento de granos, como se puede apreciar en la figura 4b).

A partir de los resultados expuestos en la figura 3 , y de acuerdo con el criterio de sensibilización utilizado para este ensayo $\left(V_{\text {corr }}>V_{\text {sen }}\right)$, se obtiene el diagrama TTS que muestra la figura 5, en el que se aprecia que la "nariz" de la curva (es decir, la temperatura para la cual se requieren menores tiempos de calentamiento para producir la sensibilización del material) se encuentra a $1.048 \mathrm{~K}$ y a tiempos menores de $1 \mathrm{~min}$. A temperaturas diferentes a la mencionada, se requieren tiempos mayores de tratamiento para producir la sensibilización.

La temperatura máxima de sensibilización es de $1.098 \mathrm{~K}$, produciéndose desensibilización para tiempos superiores a 6 min. Para temperaturas más bajas, la desensibilización se produce a tiempos mayores. El fenómeno de desensibilización a temperaturas elevadas se asocia a la redistribución de cromo como consecuencia de los altos coeficientes de difusión sustitucional del cromo a esas temperaturas o a la redisolución total o parcial de los carburos teniendo en cuenta que, al tratarse de un acero con bajo contenido de carbono, la temperatura de solubilización no debería ser muy alta y, fundamentalmente, en el caso del ensayo Huey las temperaturas de desensibilización son relativamente elevadas. 

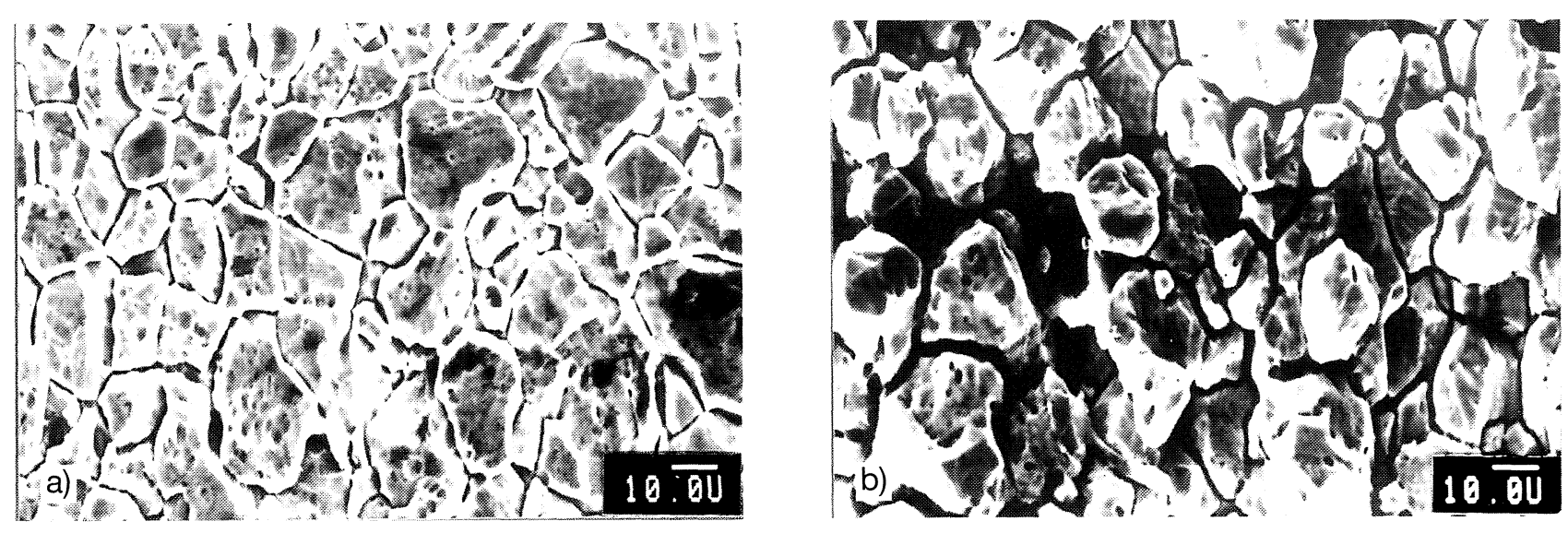

FIG. 4.- Microestructura del acero AISI 304, tratado térmicamente y expuesto al ensayo Huey:

a) $873 \mathrm{~K} / 8 \mathrm{~min}$, no sensibilizado $\left(V_{\text {corr }}=0,0025 \mathrm{~cm} / \mathrm{mes}\right) . \times 750$.

b) $973 \mathrm{~K} / 60 \mathrm{~min}$, sensibilizado $\left(V_{\text {corr }}=0,0238 \mathrm{~cm} / \mathrm{mes}\right) . \times 750$.

Fig. 4.- Microstructure of AISI 304 steel heat treated after Huey test:

a) $873 \mathrm{~K} / 8 \mathrm{~min}$, unsensitized ( $\left.\mathrm{V}_{\mathrm{corr}}=0,0025 \mathrm{~cm} / \mathrm{mes}\right) . \times 750$.

b) $973 \mathrm{~K} / 60 \mathrm{~min}$, sensitized $\left(\mathrm{V}_{\text {corr }}=0,0238 \mathrm{~cm} / \mathrm{mes}\right) . \times 750$.

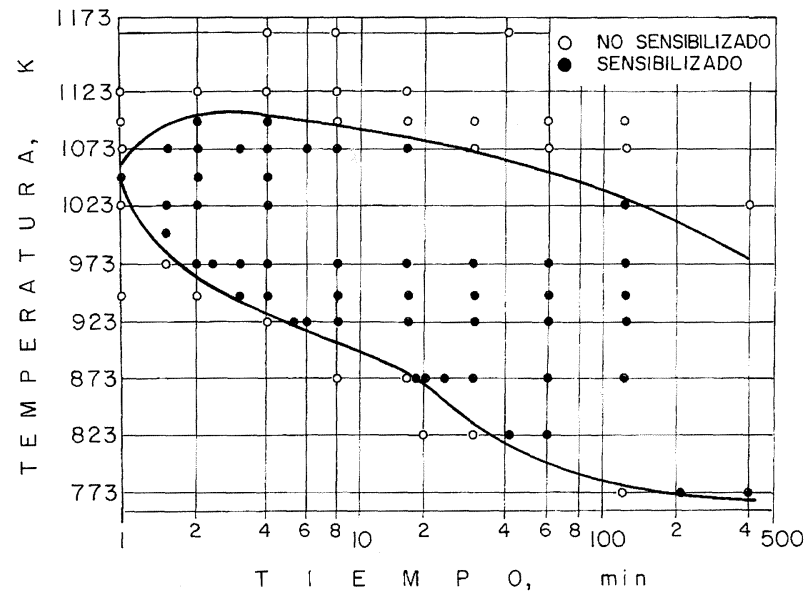

FIG. 5.- Diagrama Temperatura-Tiempo-Sensibilización (TTS) para el acero AISI 304, obtenido mediante el ensayo Huey.

Fig. 5.- Temperature-time-sensitization (TTS) diagram obtained from the Huey test for AISI 304 stainless steel.

\subsection{Resultados obtenidos mediante el ensayo Strauss modificado}

Como era de esperar, el material solubilizado, después de ensayado, no muestra síntomas de sensibilización al no presentar fisuras en la superficie de doblado, como se puede apreciar en la figura 6.

La figura 7 muestra el diagrama TTS obtenido mediante este ensayo. Se aprecia que la temperatura máxima de sensibilización está comprendida entre 943 y $985 \mathrm{~K}$. La "nariz" de la curva corresponde a $898 \mathrm{~K}$ y a un tiempo de calentamiento de $50 \mathrm{~min}$, aproximadamente. Para este ensayo, se produce también el fenómeno de desensibilización. A 943 K

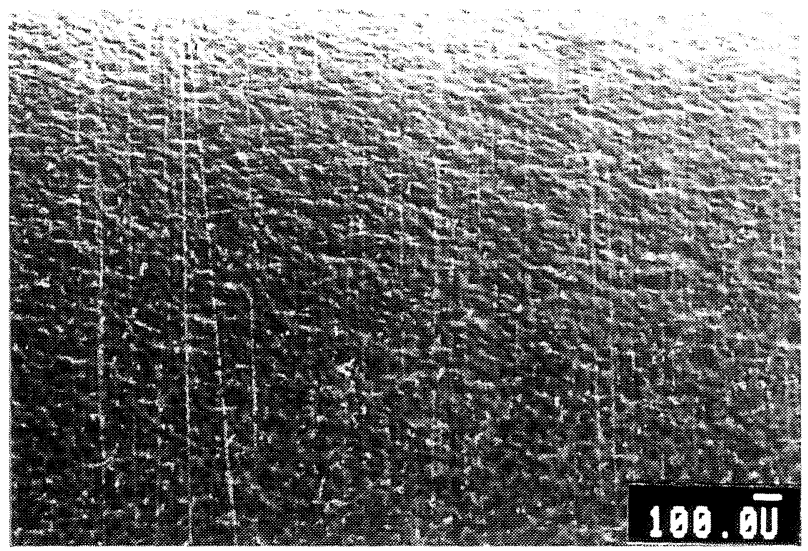

FIG. 6.- Acero AISI 304, solubilizado, sometido al ensayo Strauss modificado. Aceptable. $\times 40$.

Fig. 6.- AISI 304 steel, solubilized, after modified Strauss test. Unsensitized. $\times 40$.

y 90 min el material está sensibilizado, mientras que a tiempos mayores de 150 min el material deja de estarlo.

La figura 8a) muestra la presencia de grietas continuas en la zona de doblado, característica típica de una muestra sensibilizada a la corrosión intergranular de acuerdo con las indicaciones de este ensayo, observándose, además, (Fig. 8b) que el progreso de la grieta se produce a través de los límites de grano (grieta intergranular).

\section{COMPARACIÓN Y ANÁLISIS DE RESULTADOS}

Al comparar los resultados obtenidos para el acero AISI 304 mediante ambos ensayos 


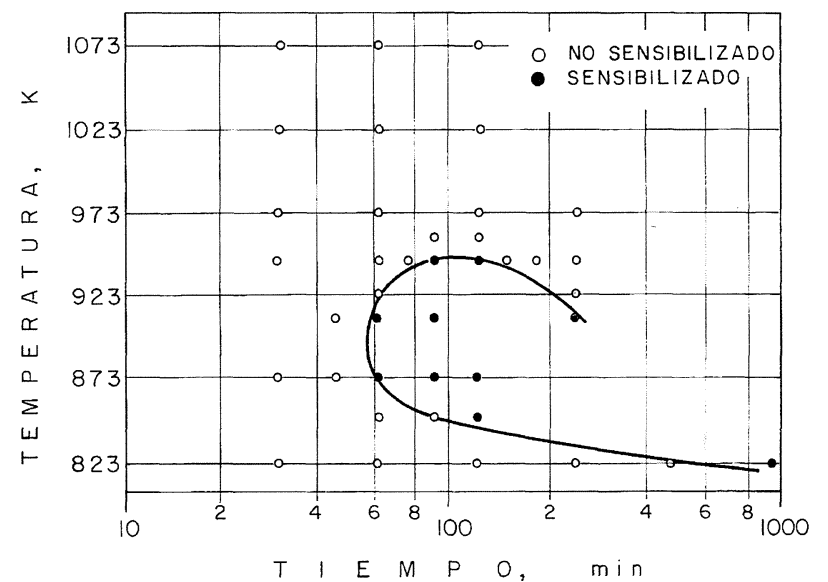

Fig. 7.- Diagrama Temperatura-Tiempo-Sensibilización (TTS) para el acero AISI 304, obtenido mediante el ensayo Strauss modificado.

Fig. 7.- TTS diagram, obtained from the modified Strauss test on AISI 304 steel.

normalizados, se puede observar, según las figuras 5 y 7 , que los diagramas obtenidos son diferentes, presentando el ensayo Huey una mayor zona de sensibilización que contiene a la del ensayo Strauss modificado. Por ello, se puede afirmar que si un material se encuentra sensibilizado de acuerdo al ensayo Strauss modificado, lo estará también en el ensayo Huey, pero no se cumplirá necesariamente lo inverso.

La temperatura, que requiere tiempos mínimos para la sensibilización, obtenida mediante el ensayo Huey es $150 \mathrm{~K}$ mayor que la obtenida para el ensayo Strauss modificado y, en cuanto al tiempo, la sensibilización se produce, para cada temperatura, a tiempos mayores para el ensayo Strauss modificado.

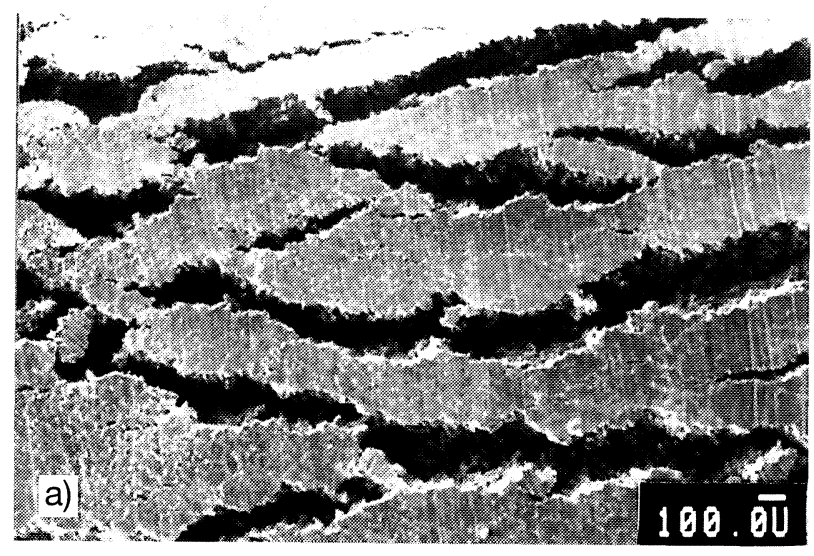

Del estudio microestructural, y a partir de las referencias mencionadas en la Introducción, se deduce que para que este material, tratado térmicamente, se encuentre sensibilizado de acuerdo con el ensayo Strauss modificado, es necesario que los carburos se agrupen e interconecten en forma de rosarios continuos, de tal manera que se produzcan bandas continuas empobrecidas en cromo en las que tendrá lugar el ataque y a través de las cuales progresará la grieta al realizar el doblado. Una configuración distinta no conducirá a la valoración positiva respecto a la sensibilización, de acuerdo con este ensayo.

En el caso del ensayo Huey, la mencionada configuración producirá las mayores $V_{\text {corr }}$, de manera que cuanto mayor sea la zona empobrecida mayores serán las velocidades de ataque, descohesionándose más rápidamente el material. $\mathrm{Al}$ aumentar la temperatura de tratamiento, la velocidad de nucleación es pequeña, debido al escaso subenfriamiento, y los carburos serán de mayor tamaño, pero no se intercomunicarán al estar separados entre sí. El material no sensible se determina al evaluarlo mediante el ensayo Strauss modificado. Respecto al ensayo Huey, el material sí se encontrará sensibilizado debido a que el electrólito empleado ataca, además de a los carburos y a las zonas empobrecidas en cromo, a una serie de precipitados localizados principalmente en límite de grano. El medio, en este caso, es mucho más agresivo que en el ensayo Strauss modificado, debido a la presencia de cationes $\mathrm{Cr}^{3+}$, que despolariza la reacción catódica ( 23 y 24$)$.

En las figuras 9a) y 9b) se observa la presencia de carburos precipitados en límites de grano en diferentes condiciones de tratamiento térmico; al evaluar el material, se observa en las curvas TTS

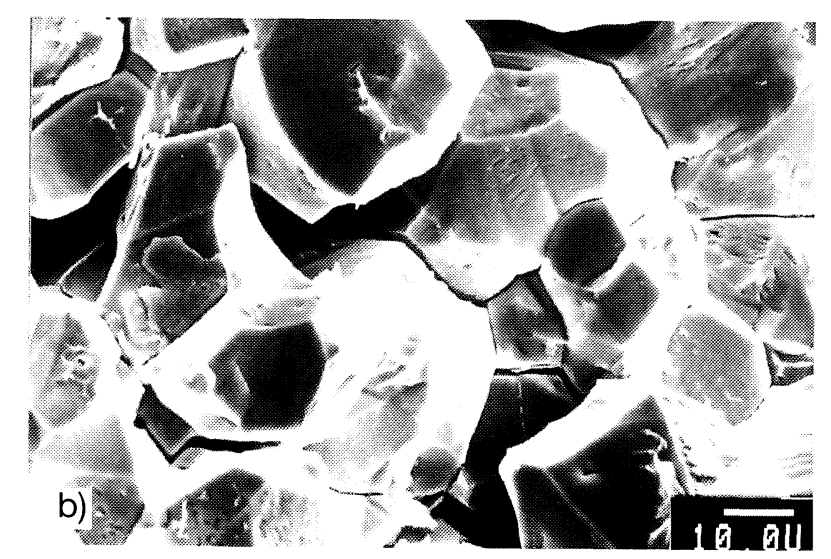

FIG. 8.- Microestructura del acero AISI 304, tratado térmicamente y expuesto al ensayo Strauss modificado: a) $823 \mathrm{~K} / 60 \mathrm{~min}$, no aceptable. $\times 40$.

b) $923 \mathrm{~K} / 30 \mathrm{~min}$, detalle de la zona de fractura. $\times 1.000$.

Fig. 8.- Microstructure of AISI 304 steel heat treated, after modified Strauss test: a) $823 \mathrm{~K} / 60 \mathrm{~min}$, sensitized. $\times 40$.

b) $923 \mathrm{~K} / 30 \mathrm{~min}$, detail of fracture way. $\times 1.000$. 

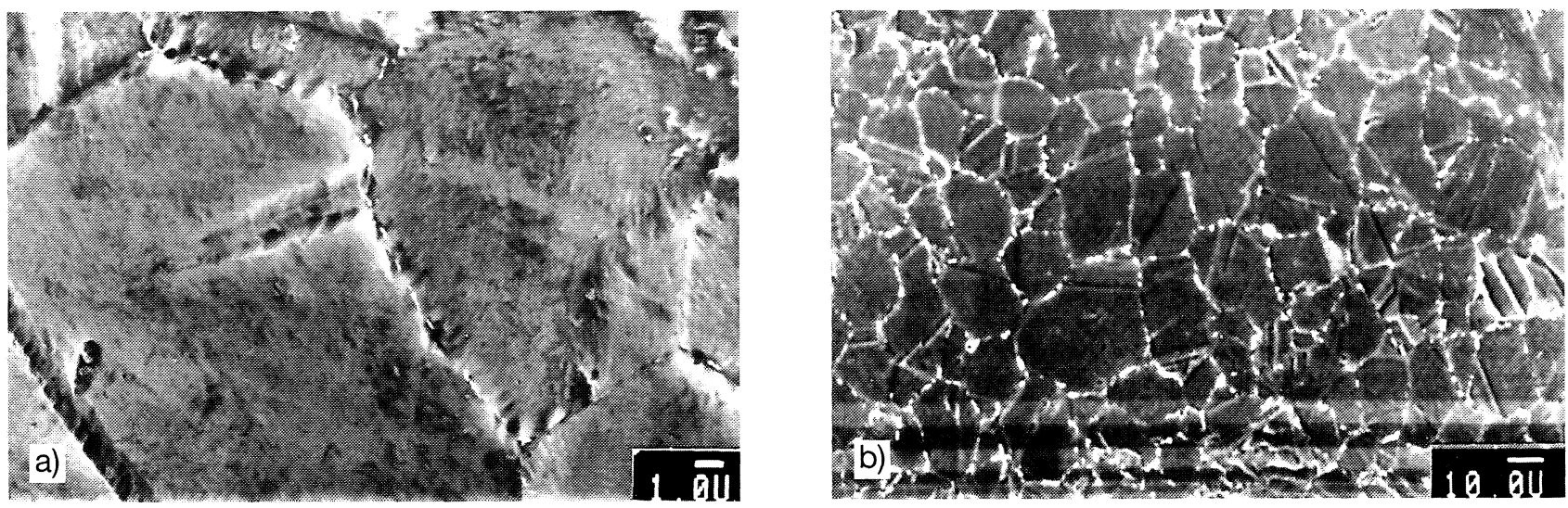

FIG. 9.- Microestructura del acero AISI 304, tratado térmicamente a:

a) $908 \mathrm{~K} / 90 \mathrm{~min} . \times 4.300$.

b) $973 \mathrm{~K} / 120 \mathrm{~min} . \times 600$.

Fig. 9.-Microstructure of AISI 304 steel heat treated to:

a) $908 \mathrm{~K} / 90 \mathrm{~min} . \times 4.300$.

b) $973 \mathrm{~K} / 120 \mathrm{~min} . \times 600$.

que para el primer caso (rosario continuo de carburos) el material se encuentra sensibilizado de acuerdo con ambos ensayos, mientras que en el segundo (partículas individuales de carburo) el material se encuentra sensibilizado con respecto al ensayo Huey.

El material ensayado presenta el fenómeno de desensibilización que se explica a patir de la difusión de cromo desde la matriz hacia las zonas empobrecidas en cromo, como consecuencia del gradiente de concentración originado por la precipitación previa del carburo. En este estudio se presenta el fenómeno de desensibilización en ambos ensayos, apreciándose que la desensibilización requiere, para el material evaluado mediante el ensayo Huey, mayores temperaturas y tiempos de tratamiento que para el material evaluado mediante el ensayo Strauss modificado.

\section{CONCLUSIONES}

Los diagramas TTS obtenidos para los materiales evaluados mediante los ensayos Huey y Strauss modificado son diferentes. Los aspectos diferenciales más relevantes son los siguientes:

- La temperatura que conduce a tiempos mínimos de sensibilización es aproximadamente $150 \mathrm{~K}$ mayor en el diagrama obtenido mediante el ensayo Huey.

- En el ensayo Huey se detecta sensibilización a menores tiempos de tratamiento térmico.

- La temperatura máxima de sensibilización es de $1.098 \mathrm{~K}$ para el ensayo Huey, y es mayor en 120 $\mathrm{K}$ a la del ensayo Strauss modificado.
Como consecuencia de lo anterior, se puede establecer que si una muestra evaluada mediante el ensayo Strauss se encuentra sensibilizada, también lo estará de acuerdo con el ensayo Huey, pero no siempre ocurre lo contrario.

La razón fundamental que justifica lo expuesto en las conclusiones anteriores, es la necesidad de la presencia de rosarios continuos de carburos interconectados para que el material dé una respuesta positiva al ensayo Strauss modificado, circunstancia que no es imprescindible que ocurra con respecto al ensayo Huey.

El fenómeno de desensibilización a altas temperaturas se presenta en ambos ensayos para el acero AISI 304. La aparición de este fenómeno requiere, para el material evaluado mediante el ensayo Huey, mayores temperaturas y tiempos de tratamiento que para el material evaluado mediante el ensayo Strauss modificado.

\section{Agradecimiento}

Los autores agradecen a la Agencia Española de Cooperación Internacional y al CICYT (proyecto MAT-0181-1993-95) la financiación de este trabajo.

\section{REFERENCIAS}

(1) Saenz, E. Tesis Doctoral. Universidad Complutense. Madrid, 1994: 97-119.

(2) Stickler, R. y Vinckier, A. Trans. Am. Soc. Met., 54 (3), 1961: 362-380.

(3) Stickler, R. y Vinckier, A. Corros. Sci., 3 (1), 1963: 1-8.

(4) KinZEL, A.B. J. Met., 4 (5), 1952: 469-488. 
(5) LEVIN, I.A. Intercrystalline corrosion and corrosion of metals under stress. Ed. Leonard Hill Ltd. Londres, 1963: 93-101.

(6) Hänninen, H.B. Int. Met. Rev., 24 (3), 1979: 85-135.

(7) Abe, S. y Kaneko, M. 11 th Intern. Corrosion Cong. Associazione Italiana di Metallurgia. Florencia (Italia), 1990: 5.195-5.202.

(8) Abe, S. y KaneKo, M. Stainless Steel '91. Iron and Steel Institute of Japan. Vol. I. Chiba (Japón), 1991: 272-279.

(9) Bain, E.C., Aborn, R.H. y Rutherford, J. Trans. Am. Soc. Met., 21 (3), 1933: 481-509.

(10) Gelling, P.J. Corros. Sci., 7 (6), 1967: 413-421.

(11) Briant, C.L. Metall. Trans., 18A (4), 1987: 691-699.

(12) Briant, C.L. y Andressen, P.L. Metall. Trans., 19A (3), 1988: 495-504.

(13) Joshi, A. y Stein, J.F. Corrosion (Houston), 28 (9), 1972: 321-330.

(14) Aho-Mantilla, I., Aaltonen, P. y Hänninen, H. Intergranular corrosion testing of austenitic stainless steels. Technical Research Centre of Finland, Research Report 26/1981.
(15) RaO, K.P. Tool Alloy Steel, 19 (9), 1985: 289-296.

(16) WALKER, W.L. Intergranular corrosion of stainless alloys Ed. R.F. Steigerwald. ASTM 656. Filadelfia (EE.UU.), 1978: 146-153.

(17) CoRbett, R.A. Corrosion testing and evaluation, ASTM. Orlando, Florida (EE.UU.), 1989: 335-347

(18) ASTM Standard A262-86, practice C. Standard practices for detecting susceptibility to intergranular attack in austenitic stainless steel.

(19) Fontana, M.G. y GreEne, M.D. Corrosion Engineering. Ed. Mc Graw Hill. Nueva York, 1962: 53.

(20) DuPont Standard. SW 800 (rev. Ago. 1985). Corrosion evaluation of stainless steels and nickel-base alloys.

(21) ASTM Standard A262-86, practice E. Standard practices for detecting susceptibility to intergranular attack in austenitic stainless steel.

(22) Scharfstein, L.R. y Eisenbrown, M.C. ASTM STP 369, ASTM. Filadelfia (EE.UU.), 1963: 235-239.

(23) Nagano, H. Corros. Eng., 37 (5), 1988: 263-274.

(24) Kajimura, H., Morikawa, H. y Nagano, H. Corros. Eng., 36 (10), 1987: 579-586. 